\title{
Raman Polarization Pulling and Amplification for Polarization Division Multiplexing Transmission System
}

\author{
Krzysztof Perlicki
}

\begin{abstract}
The numerical modelling of the Raman polarization attraction and amplification process in the telecommunication band around $1550 \mathrm{~nm}$ is presented. The possibility of achieving both polarization pulling and amplification in Polarization Division Multiplexing transmission system by exploiting the polarization dependence of stimulated Raman scattering is investigated. The acceptable crosstalk for the Polarization Division Multiplexing transmission systems was achieved for some attraction/amplification block parameters.
\end{abstract}

Keywords-Fiber optics transmsission, light polarization, polarization division multiplexing, Raman scattering, polarization pulling.

\section{INTRODUCTION}

$\mathbf{T}$ HE interest towards multifunction optical phenomena or multifunction optical devices should be stimulated in the near future. For example, the stimulated Raman scattering effect can be used for combination of a polarization controlling and optical amplication. The stimulated Raman scattering is an optical phenomenon that recently has had commercial interest because of its potential technologies in engineering. The stimulated Raman scattering has become important in the application of optical amplification because of several important reasons in comparison to other amplification [1]. Additionally, stimulated Raman scattering can be used for polarization attraction effect [2], [3]. In real fibers the input states of polarization (SOPs) are not preserved because of the random birefringence (polarization mode dispersion). The uncontrolled SOPs variable can dramatically affect the performances of telecommunication systems. This phenomenon is very important, especially for Polarization Division Multiplexing (PDM) transmission system. In this system two independently modulated data channels with the same wavelength, but orthogonal SOPs are simultaneously transmitted in a single optical fiber. Two orthogonally polarized signals are transmitted to double the data throughput. At the receiver end, the two polarization channels are separated by polarization beam splitter and detected independently. The SOP for output optical signal should be linear $+45^{\circ}$ [4]. For this SOP value the demultiplexing proccess is correct and crosstalk between polarization channels is neglected. However, separating the two polarization channels with acceptable crosstalk (less than

K. Perlicki is with Orange Labs, Research and Development Centre Branch, Telekomunikacja Polska, Obrzeżna 7, 02-691 Warsaw, Poland (email: Krzysztof.Perlicki@telekomunikacja.pl) and with Institute of Telecommunications, Warsaw University of Technology, Nowowiejska 15/19, 00-665 Warsaw, Poland (phone: +48 22 2347464, e-mail: perlicki@tele.pw.edu.pl).
$-20 \mathrm{~dB})$ at the receiving end is not trivial, because the polarization states of the two channels change rapidly with time [5]. This problem can be solved by means of polarization attraction effect. The polarization pulling, which is based on stimulated Ramana scattering effect, can be implemented for the polarization stabilization of PDM transmission system. In the present paper the possibility of achieving both polarization pulling and amplification in PDM transmission system by exploiting the polarization dependence of stimulated Raman scattering is investigated. The conditions for obtaning proper polarization pulling and optical signal amplification by exploiting the stimulated Raman scattering are investigated. The optimal conditons for polarization pulling and Raman amplification for PDM transmission system are analysed.

\section{TheORETICAL BACKGROUND}

The conceptual diagram of polarization attraction/amplification block scheme for PDM transmission system is shown in Fig. 1. Optical data streams TX1 and TX2 with orthogonal SOPs (LH - linear horizontal, LV linear vertical) are generated at the same wavelength and then multiplexed through a polarization beam combiner (PBC). During signal transmission over a optical fiber transmission link (FOL), the signal SOP evolves into an aritrary polarization. The output signal SOP at the point $\mathrm{A}$ is arbitrary. The attraction/amplification block (AAB) followed by a polarization beam splitter (PBS) is used to polarization pulling and output signal amplification. The output signal SOP should be linear $+45^{\circ}$ at the point B. Next, the two polarization channels are separated by PBS. The polarization attraction/amplification block (AAB) consisits of: laser pump (LP), polarization controller (PC) and single mode optical fiber link (FO). The values of attraction/amplification block parameters (i.e.: pump power, pump SOP and optical fiber link length) should be accurately selected depending on expected polarization pulling and Raman gain. The Raman gain is defined as the ratio of the power of the signal with and without Raman amplification. The polarization pulling quality is assessed by the crosstalk value between polarization channels. The crosstalk is difference between proper signal and crosstalk signal from adjacent polarization channel [6]. The power evolution of the pump (P) and signal $(\mathbf{S})$ for copumped configuration along the optical fiber link FO can be modeled by means of coupled equations, respectively [7]:

$$
\frac{d \mathbf{P}}{d z}=-\alpha_{p} \mathbf{P}-\frac{\omega_{p}}{2 \omega_{s}} g_{R}\left(P_{0} \mathbf{S}+S_{0} \mathbf{P}\right)+\left(\omega_{p} \mathbf{b}+\mathbf{W}_{P}^{\mathbf{N L}}\right) \times \mathbf{P}
$$




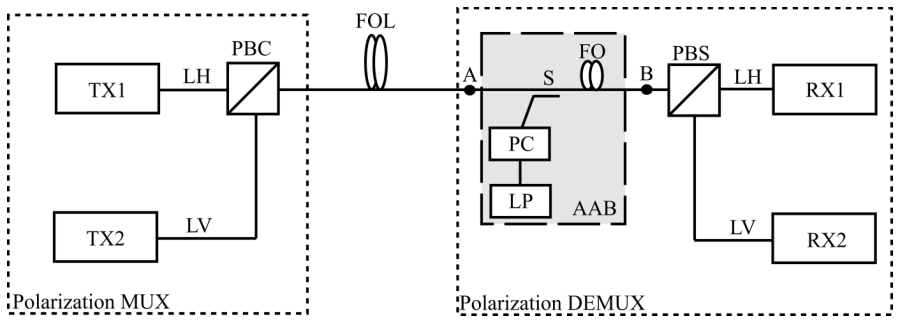

Fig. 1. Diagram of proposed polarization DEMUX using stimulated Ramana scattering effect. TX1, TX2 - transmitters, LH - linear horizontal polarization, LV - linear vertical polarization, PBC - polarization beam combiner, FOL optical fiber transmission link, AAB - attraction/amplification block, LP Raman laser pump, PC - polarization controller, FO - optical fiber link for Raman amplification, $\mathrm{S}$ - splitter, PBS - polarization beam splitter, RX1, RX2 - receivers.

$$
\frac{d \mathbf{S}}{d z}=-\alpha_{s} \mathbf{S}+\frac{1}{2} g_{R}\left(S_{0} \mathbf{P}+P_{0} \mathbf{S}\right)+\left(\omega_{s} \mathbf{b}+\mathbf{W}_{S}^{\mathbf{N L}}\right) \times \mathbf{S},
$$

where $\omega_{p}$ and $\omega_{s}$ are the pump and signal carrier angular frequencies, $\alpha_{p}$ and $\alpha_{s}$ are the optical fiber FO attenuation coefficients for the pump and signal wavelengths, respectively. The $g_{R}$ component is the Raman gain coefficient. The vector lengths $P_{0}=|\mathbf{P}|$ and $S_{0}=|\mathbf{S}|$ represent the pump and signal powers, respectively. The vector $\mathbf{b}$ is the local linear birefringence for the optical fiber FO. The linear birefringence vector used in the numerical solutions was derived from the Random Modulus Model [3]. In turn, the vectors $\mathbf{W}_{P}^{\mathbf{N L}}$ and $\mathbf{W}_{S}^{\mathbf{N L}}$ are given by [2]:

$$
\begin{aligned}
& \mathbf{W}_{P}^{\mathbf{N L}}=\frac{2}{3} \gamma_{p}\left(-2 S_{S, 1},-2 S_{S, 2},-S_{P, 3}\right), \\
& \mathbf{W}_{S}^{\mathbf{N L}}=\frac{2}{3} \gamma_{s}\left(-2 S_{P, 1},-2 S_{P, 2},-S_{S, 3}\right),
\end{aligned}
$$

where $\gamma_{p}$ and $\gamma_{s}$ are the nonlinear Kerr coefficients, $S_{P, 1}, S_{P, 2}$, $S_{P, 3}, S_{S, 1}, S_{S, 2}, S_{S, 3}$ are the Stokes parameters for the pump and signal, respectively.

\section{NUMERICAL RESULTS}

The equations (1) and (2) were numerically solved by a Runge-Kutta method of $4^{\text {th }}$ order. The following simulation conditions were assumed. Optical signals parameters:

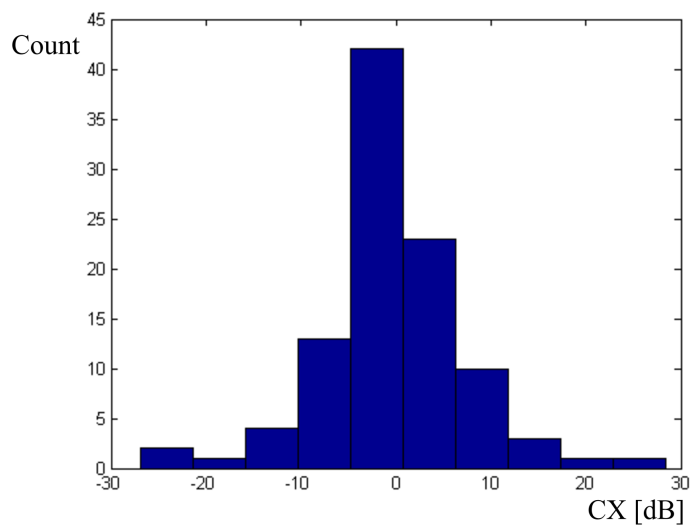

Fig. 2. Histogram of crosstalk values for PDM system without attraction/amplification block.
TABLE I

The INPUt Pump SOP FOR the Signal Polarization CONVERges to LINEAR $+45^{\circ}$ POLARIZATION

\begin{tabular}{c|ccc}
\hline \hline & $P_{0}(0)=1 \mathrm{~W}$ & $P_{0}(0)=2 \mathrm{~W}$ & $P_{0}(0)=5 \mathrm{~W}$ \\
\cline { 2 - 4 } & $S_{1}, S_{2}, S_{3}$ & $S_{1}, S_{2}, S_{3}$ & $S_{1}, S_{2}, S_{3}$ \\
\hline $\mathrm{L}_{F O}=1 \mathrm{~km}$ & $0.1450,-0.8035$, & $0.9712,0.2108$, & $0.5269,0.8449$, \\
& -0.5773 & -0.1115 & -0.0920 \\
$\mathrm{~L}_{F O}=2 \mathrm{~km}$ & $-0.5417,0.1460$, & $0.4576,0.4963$, & $0.7803,0.1264$, \\
& -0.8278 & 0.7378 & 0.6125 \\
$\mathrm{~L}_{F O}=5 \mathrm{~km}$ & $0.8343,-0.0384$, & $0.2239,0.4745$, & $0.9992,-0.0143$, \\
& $-0,5499$ & -0.8513 & 0.0372 \\
$\mathrm{~L}_{F O}=10 \mathrm{~km}$ & $-0.1944,0.8326$, & $0.9872,-0.1056$, & $0.6019,0.4503$, \\
& 0.5186 & -0.1194 & -0.6595 \\
\hline \hline
\end{tabular}

1) The pump and signal angular frequencies are $\omega_{p}=2 \pi c / \lambda_{p} \quad\left(\lambda_{p}=1450 \mathrm{~nm}\right)$ and $\omega_{s}=2 \pi c / \lambda_{s}$ $\left(\lambda_{s}=1550 \mathrm{~nm}\right)$; where $c$ is the vacuum speed of light.

2) The signal power at the point $\mathrm{A}$ is set to $10 \mu \mathrm{W}(-20$ $\mathrm{dBm})$.

Optical fiber link FO parameters:

1) The attenuation coeffcients for the pump and signal wavelengths are $\alpha_{p}=0.273 \mathrm{~dB} / \mathrm{km}$ and $\alpha_{s}=0.2$ $\mathrm{dB} / \mathrm{km}$, respectively.

2) The Raman gain coefficient $g_{R}=0.6 \mathrm{~W}^{-1} \mathrm{~km}^{-1}$.

3) The nonlinear Kerr coefficients $\gamma_{p}=1.24 \mathrm{~W}^{-1} \mathrm{~km}^{-1}$ and $\gamma_{s}=1.06 \mathrm{~W}^{-1} \mathrm{~km}^{-1}$.

4) The beat length $L_{B}=44 \mathrm{~m}$, the correlation length $L_{c}=340 \mathrm{~m}$ and the polarization mode dispersion coefficient $D_{P M D}=0.1 \mathrm{ps} / \sqrt{\mathrm{km}}$.

We assumed that the environmental conditions for the polarization attraction/amplification block are stationary. Therefore the same birefringence realization was taken into account. At the beginning, for a single arbitrarily polarized signal at the point A (Fig. 1) the input pump SOP was searched for the signal polarization pulling towards linear $+45^{\circ}$ polarization at

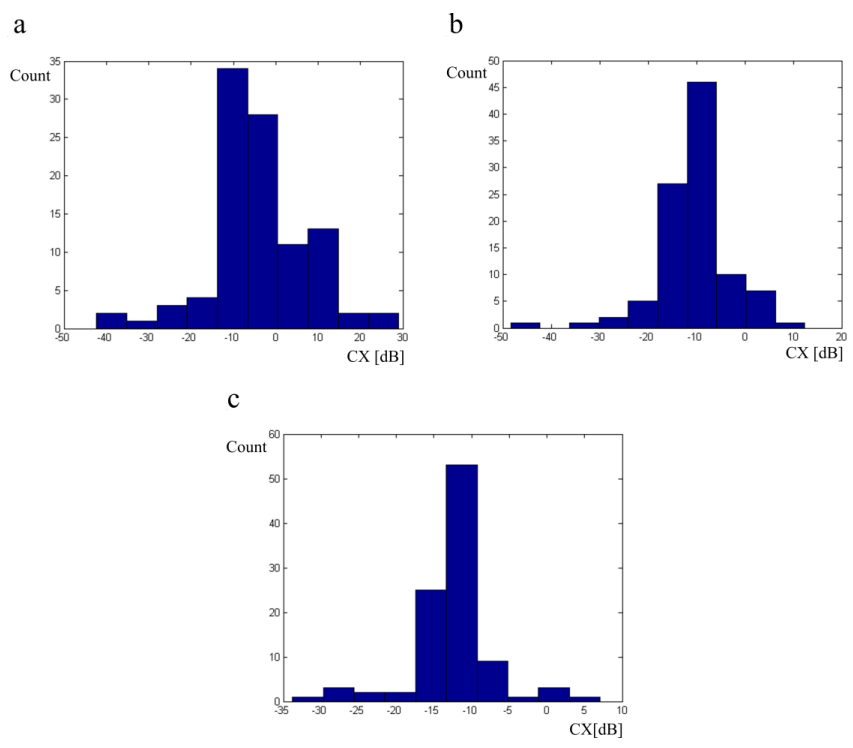

Fig. 3. Histogram of crosstalk values for PDM system with attraction/amplification block for $\mathrm{L}_{F O}=1 \mathrm{~km}, \mathrm{a}-P_{0}(0)=1 \mathrm{~W}$ and input pump SOP $(0.1450,-0.8035,-0.5773), \mathrm{b}-P_{0}(0)=2 \mathrm{~W}$ and input pump SOP $(0.9712,0.2108,-0.1115), \mathrm{c}-P_{0}(0)=5 \mathrm{~W}$ and input pump SOP $(0.5269$, $0.8449,-0.0920)$. 

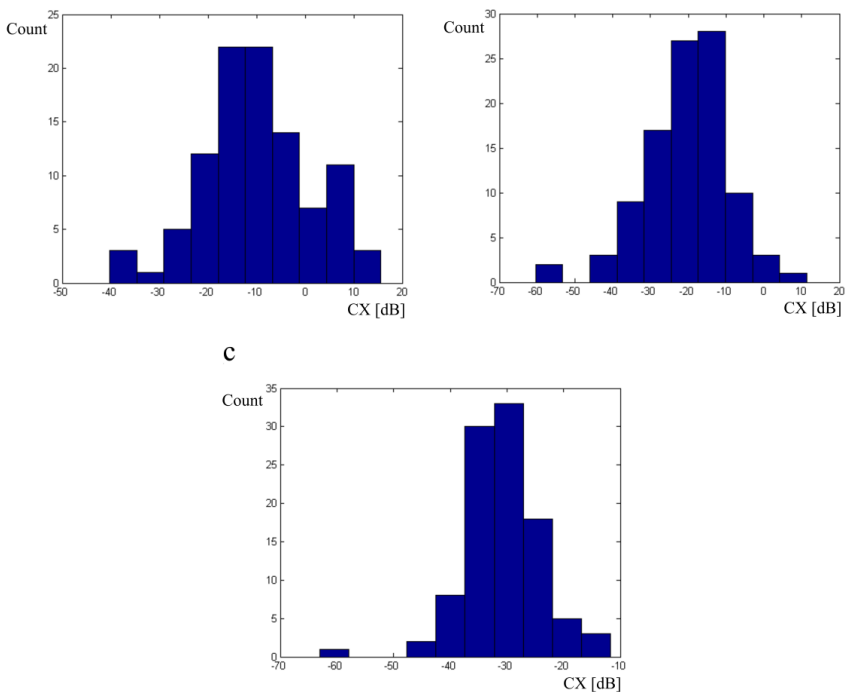

Fig. 4. Histogram of crosstalk values for PDM system with attraction/amplification block for $\mathrm{L}_{F O}=2 \mathrm{~km}, \mathrm{a}-P_{0}(0)=1 \mathrm{~W}$ and input pump SOP $(-0.5417,0.1460,-0.8278), \mathrm{b}-P_{0}(0)=2 \mathrm{~W}$ and input pump SOP $(0.4576,0.4963,0.7378), \mathrm{c}-P_{0}(0)=5 \mathrm{~W}$ and input pump SOP $(0.7803$, $0.1264,0.6125)$

the point B (Fig. 1).

The proper value of input pump SOP was searched by algorithm which describes distributing of SOPs uniformly over Poincare sphere [8]. The input pump SOP can be changed by means of polarization controller (PC). Different pump (LP) power and optical fiber link (FO) length values were taken into account. The numerical simulations were performed for input pump power $P_{0}(0)$ and optical fiber link length $\mathrm{L}_{F O}$ which were equal to $1 \mathrm{~W}, 2 \mathrm{~W}, 5 \mathrm{~W}$ and $1 \mathrm{~km}, 2 \mathrm{~km}, 5 \mathrm{~km}, 10 \mathrm{~km}$, respectively. Table I shows the input pump Stokes parameters
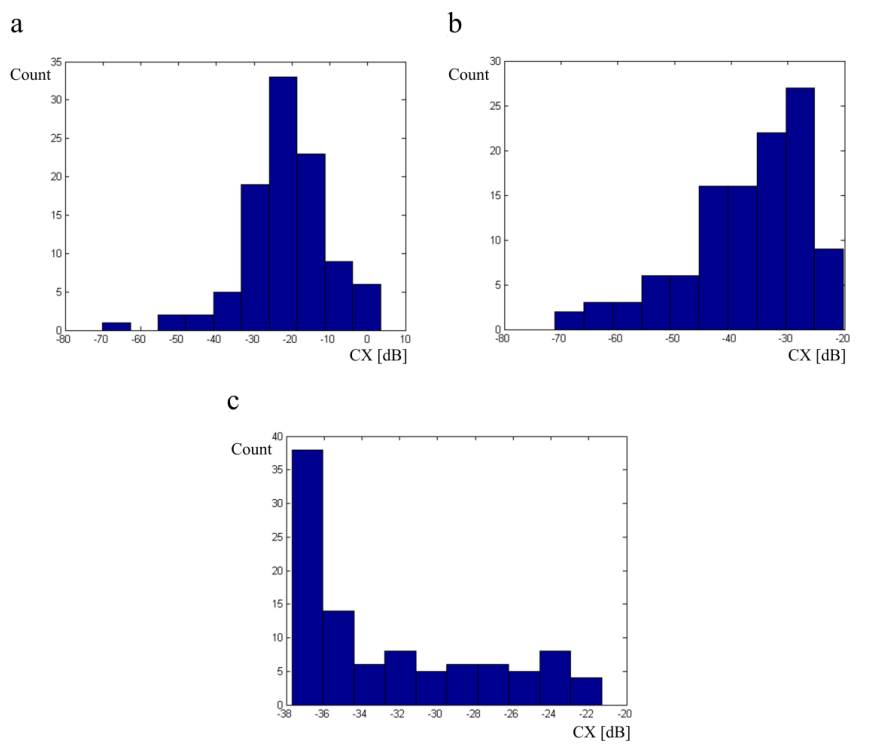

Fig. 5. Histogram of crosstalk values for PDM system with attraction/amplification block for $\mathrm{L}_{F O}=5 \mathrm{~km}, \mathrm{a}-P_{0}(0)=1 \mathrm{~W}$ and input pump SOP $(0.8343,-0.0384,-0,5499), \mathrm{b}-P_{0}(0)=2 \mathrm{~W}$ and input pump SOP $(0.2239,0.4745,-0.8513), \mathrm{c}-P_{0}(0)=5 \mathrm{~W}$ and input pump SOP $(0.9992,-0.0143,0.0372)$.

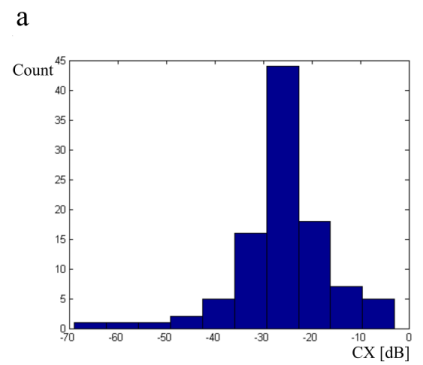

b

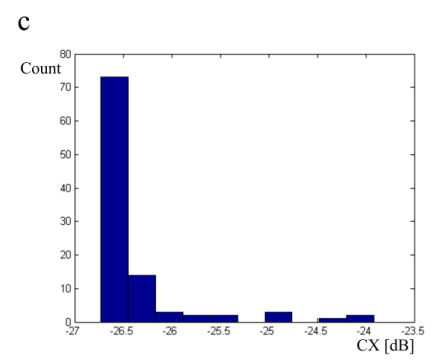

Fig. 6. Histogram of crosstalk values for PDM system with attraction/amplification block for $\mathrm{L}_{F O}=10 \mathrm{~km}, \mathrm{a}-P_{0}(0)=1 \mathrm{~W}$ and input pump SOP $(-0.1944,0.8326,0.5186), \mathrm{b}-P_{0}(0)=2 \mathrm{~W}$ and input pump SOP $(0.9872,-0.1056,-0.1194), \mathrm{c}-P_{0}(0)=5 \mathrm{~W}$ and input pump SOP $(0.6019$, $0.4503,-0.6595)$.

$\left(S_{1}, S_{2}, S_{3}\right)$ for achieving of linear $+45^{\circ}$ signal polarization at the point B.

Next the quality of polarization pulling was assessed by the calculation of the polarization crosstalk. The crosstalk values CX were calculated for 100 arbitrary signal SOPs at the point A. Figure 2 shows the histogram of crosstalk values for PDM system without the attraction/amplification block.

Figures 3, 4, 5 and 6 show the histograms of crosstalk values for PDM system with the attraction/amplification block. The presented results were obtained for $\mathrm{L}_{F O}=1 \mathrm{~km}$ (Fig. 3), $\mathrm{L}_{F O}=2 \mathrm{~km}$ (Fig. 4), $L_{F O}=5 \mathrm{~km}$ (Fig. 5), $\mathrm{L}_{F O}=10 \mathrm{~km}$ (Fig. 6) and $P_{0}(0)=1 \mathrm{~W}, 2 \mathrm{~W}, 5 \mathrm{~W}$.

Figures 7 and 8 show simulated examples of polarization pulling effect for $\mathrm{L}_{F O}=5 \mathrm{~km}$ and $P_{0}(0)=1 \mathrm{~W}, 2 \mathrm{~W}$ and 5 W.

As can be seen, when the pump power increases the output signal SOPs collapse to particular, preffered state. The output signal SOPs converge to linear $+45^{\circ}$ polarization. The acceptable crosstalk for PDM system $(\mathrm{CX}<-20 \mathrm{~dB})$ was obtained

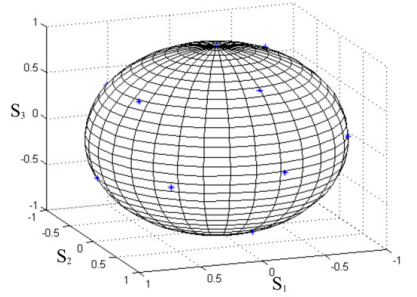

b

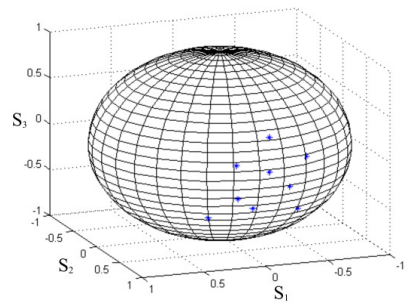

Fig. 7. Simulated examples of polarization pulling; a - distrubution of arbitrarily polarized signals at the point $\mathrm{A}(\mathrm{a}), \mathrm{b}-$ distrubution of output signal SOPs at the point $\mathrm{B}$ for $\mathrm{L}_{F O}=5 \mathrm{~km}$ and $P_{0}(0)=1 \mathrm{~W}$ and input pump SOP (0.8343, -0.0384, -0,5499). 

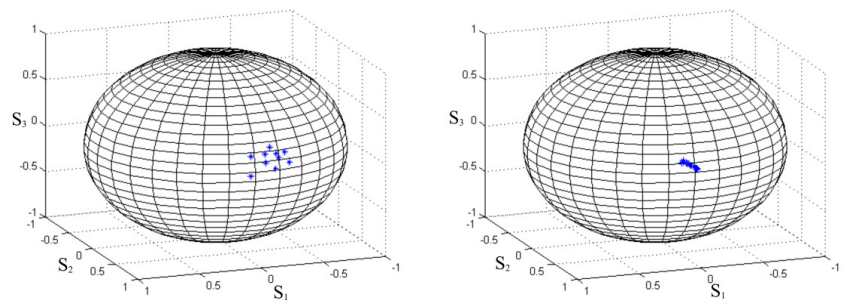

Fig. 8. Simulated examples of polarization pulling; a - distrubution of output signal SOPs at the point $\mathrm{B}$ for $\mathrm{L}_{F O}=5 \mathrm{~km}$ and $P_{0}(0)=2 \mathrm{~W}$ and input pump SOP $(0.2239,0.4745,-0.8513), \mathrm{b}$ - distrubution of output signal SOPs at the point B for $L_{F O}=5 \mathrm{~km}$ and $P_{0}(0)=5 \mathrm{~W}$ and input pump SOP $(0.9992,-0.0143,0.0372)$
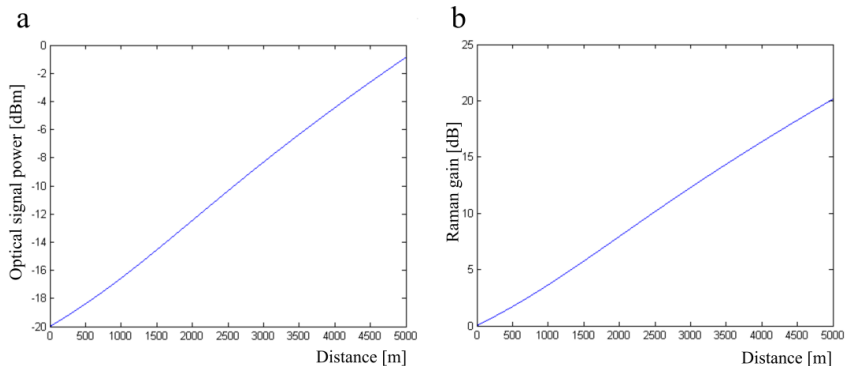

Fig. 9. Signal power evolution (a) and Raman gain evolution (b) along the optical fiber link FO; $\mathrm{L}_{F O}=5 \mathrm{~km}$ and $P_{0}(0)=2 \mathrm{~W}$, input pump SOP $(0.2239,0.4745,-0.8513)$, arbitrary signal SOP at the point A.

for $\mathrm{L}_{F O}=5 \mathrm{~km}$ or $10 \mathrm{~km}$ and $P_{0}(0)=2 \mathrm{~W}$ or $P_{0}(0)=5 \mathrm{~W}$. We can find commercially available Raman laser pump with optical power up $2 \mathrm{~W}$. This value is still dangerless for optical fiber links, especially for optical components (e.g. connectors). The pump with $5 \mathrm{~W}$ power should be treated as theoretical approach. Furthermore the optical fiber link FO length of $5 \mathrm{~km}$ is more user friendly than $10 \mathrm{~km}$. Therefore we can assume that the optimal conditons of the AAB block for the polarization pulling function are: pump power of $2 \mathrm{~W}$, input pump SOP $(0.2239,0.4745,-0.8513)$ and length of the opical fiber link FO equals to $5 \mathrm{~km}$. Next, the power and Raman gain evolution for the above mentioned conditions were calculated. The power evolution of the signal along optical fiber link FO is shown in Fig. 9a. In turn, Fig. 9b shows the evolution of the distributed Raman gain along optical fiber link FO.

As can be seen in Fig. 9, the values of optical signal power and Raman gain which were obtained by means of attraction/amplification block are appropriate for telecommunication applications.

\section{CONCLUSION}

In this work the numerical modelling of the Raman polarization attraction process in the telecommunication band around $1550 \mathrm{~nm}$ was presented. In conclusion, the proper Raman polarization pulling and amplification for PDM systems can be simultaneously achieved. The acceptable crosstalk for the PDM systems was achieved for some attraction/amplification block parameters. The best polarization pulling phenomenon was observed for $\mathrm{L}_{F O}=5 \mathrm{~km}$ or $10 \mathrm{~km}$ and $P_{0}(0)=2 \mathrm{~W}$ or $P_{0}(0)=5 \mathrm{~W}$. Nevertheless, I would like to emphasize that a critical challenge in the present system here is that high pump power is required. The very highly nonlinear fibers could be used so that the required powers for Raman polarization attraction process may be compatible with usual telecommunication parameters.

\section{REFERENCES}

[1] G. P. Agrawal, Fiber-OpticCommunications Systems. New York: Wiley, 2002.

[2] M. Martinelli, M. Cirigliano, M. Ferrario, L. Marazzi, and P. Martelli, "Evidence of Raman-induced Polarization Pulling," Optics Express, vol. 17, no. 2, pp. 947-955, 2009.

[3] A. Galtarossa, L. Palmieri, M. Santagiustina, and L. Ursini, "Polarized Backward Raman Amplification in Randomly Birefringent Fibers," Journal of Lightwave Technology, vol. 24, pp. 4055-4063, 2006.

[4] X. S. Yao, "All-optic Scheme for Automatic Plarization Division Demultiplexing," Optics Express, vol. 15, no. 12, pp. 7407-7414, 2007.

[5] D. van den Borne, N. E. Hecker-Denschlag, and G. D. Khoe, "PMD Induced Transmission Penalties in Polarization-Multiplexed Transmission," Journal of Lightwave Technology, vol. 23, no. 12, pp. 4004-4014, 2005.

[6] E. Rochat, S. D. Walker, and M. C. Parker, "Polarisation and Wavelength Division Multiplexing at $1.55 \mu \mathrm{m}$ for Bandwidth Enhancement of Multimode Fibre Based Access Networks," Optics Express, vol. 17, no. 10, pp. 2280-7414, 2004.

[7] Q. Lin and G. P. Agrawal, "Statistics of Polarization-dependent Gain in Fiber-based Raman Amplifiers," Optics Letters, vol. 28, no. 4, pp. 227 229, 2003.

[8] E. B. Saff, "Distributing Many Pints on a Sphere," The Mathematical Intelligencer, vol. 19, no. 1, pp. 5-11, 1997. 